\title{
Decellularized versus standard cryopreserved valve allografts for right ventricular outflow tract reconstruction: A single-institution comparison
}

\author{
Mark Ruzmetov, MD, PhD, Jitendra J. Shah, MD, Dale M. Geiss, MD, and Randall S. Fortuna, MD
}

\begin{abstract}
Background: Standard cryopreserved valved allografts (SCAs) are recognized as the benchmark for reconstruction of the right ventricular outflow tract (RVOT). However, SCAs frequently demonstrate early valve deterioration and elicit an immune response. Decellularized cryopreserved valve allografts (SynerGraft, SG) are less immunogenetic and may be more durable. This study analyzed our results of RVOT reconstruction using SGs and compared it with the SCAs used during the same period.
\end{abstract}

Methods: We reviewed the outcome of all allografts (SG and SCA) that were implanted for RVOT reconstruction at a single center from 2000 to 2005 . Echocardiographic data were reviewed to evaluate valve performance. Conduit failure is defined as the need for conduit replacement or reintervention in either the catheterization laboratory or operating room. Conduit dysfunction is defined as RVOT obstruction with peak echocardiographic Doppler gradient greater than $40 \mathrm{~mm} \mathrm{Hg}$ and/or grade III/IV or greater conduit valve regurgitation. Data were compared using the Wilcoxon rank sum and Fisher's exact test.

Results: From January 2000 to April 2005, 100 patients (mean age $18.6 \pm 16.8$ years) received SG ( $\mathrm{n}=39$ ) or SCA $(n=61)$ conduits. The 2 retrospective nonrandomized cohorts were similar with respect to age, gender, weight, conduit indication, bypass and crossclamp time, and conduit size. Follow-up time was not significant between the 2 groups (SG, $5.7 \pm 2.5$ years vs SCA, $5.8 \pm 2.8$ years; $P=.83$ ). Early and late mortality were similar (SG, $13 \%$; SCA, $10 \% ; P=.75$ ). No death was graft related. Freedom from dysfunction was superior with SG (SG, 74\%, vs SCA, 52\%; $P=.05$ ). Freedom from failure was also better in patients with SG (SG, $87 \%$, vs SCA, $68 \% ; P=.05$ ). Freedom from explantation and more than moderate pulmonary insufficiency were significantly better for SG patients (SG, $92 \%$ and $90 \%$, vs SCA, $78 \%$ and $68 \% ; P=.02$ ).

Conclusions: This study suggests that the midterm performance of SGs may be superior to that of SCAs. Decellularization of the cryopreserved allografts may provide a more durable option for patients who need RVOT reconstruction. Further long-term follow-up is needed to see whether this decellularization process improves long-term allograft durability. (J Thorac Cardiovasc Surg 2012;143:543-9)

Right ventricular-pulmonary artery (RV-PA) conduits have made possible the repair of many complex congenital cardiac lesions involving atresia or hypoplasia of the RV outflow tract (RVOT). These fundamental diagnoses include truncus arteriosus, pulmonary atresia with ventricular septal defect, severe tetralogy of Fallot, transposition with ventricular septal defect and pulmonary atresia, and various forms of doubleoutlet RV. Conduits have also made possible the pulmonary autograft replacement of the aortic root (Ross procedure). ${ }^{1}$

\footnotetext{
From the Children Hospital of Illinois, OSF Saint Francis Medical Center, and University of Illinois College of Medicine at Peoria, Peoria, Ill.

Disclosures: This study was partially funded by an unrestricted research grant from CryoLife, Inc.

Read at the 37th Annual Meeting of The Western Thoracic Surgical Association, Colorado Springs, Colorado, June 22-25, 2011.

Received for publication June 6, 2011; revisions received Oct 18, 2011; accepted for publication Dec 14, 2011.

Address for reprints: Randall S. Fortuna, MD, Children Hospital of Illinois, OSF Saint Francis Medical Center, 515 NE Glen Oak Ave, Suite 202, Peoria, IL 63603 (E-mail: rfortuna@ilcardiac.com).

$0022-5223 / \$ 36.00$

Copyright (c) 2012 by The American Association for Thoracic Surgery doi:10.1016/j.jtcvs.2011.12.032
}

Numerous valved conduits have been introduced since an aortic allograft was first used clinically in the mid-1960s. ${ }^{2-5}$ Conduit types include bioprostheses in Dacron tubes, stented bovine or porcine xenografts in pericardial tubes, glutaraldehyde-fixed aortic or pulmonary roots, nonvalved tissue or prosthetic conduits, and aortic and pulmonary allografts.

The standard cryopreserved allograft (SCA) subsequently became the conduit of choice in the United States for RVOT reconstruction in the mid-1980s. Development of cryopreservation techniques has improved the availability and durability of allografts considerably, resulting in increasing use in clinical practice. Early results with SCAs for reconstruction of the RVOT in congenital heart disease have been good, but there are few long-term studies reporting allograft dysfunction and failure. ${ }^{4-6}$

Many SCAs are prone to shrinkage, leading to conduit stenosis and the development of significant valve regurgitation within months of insertion, especially when implanted as extracardiac conduits. ${ }^{5}$ In most patients receiving a transplanted allograft valve, humoral antibodies develop against human 


$$
\begin{aligned}
& \text { Abbreviations and Acronyms } \\
& \begin{aligned}
\text { PA } & =\text { pulmonary artery } \\
\text { PRA } & =\text { panel reactive antibody } \\
\text { RVOT } & =\text { right ventricular outflow tract } \\
\text { RV } & =\text { right ventricle (ventricular) } \\
\text { SCA } & =\text { standard cryopreserved allograft } \\
\text { SG } & =\text { SynerGraft }
\end{aligned}
\end{aligned}
$$

leukocyte antigen that are specific to the transplanted tissue, ${ }^{7}$ and host antigen recognition and antibody development may be linked to early-onset tissue calcification and structural valve deterioration. ${ }^{8-10}$ To improve these shortcomings, the SynerGraft (SG; CryoLife, Inc, Kennesaw, Ga) process decellularizes an allograft, leaving only connective tissue, which then may become repopulated with host cells. ${ }^{10}$

Similar to the SCA, SG allografts do not need anticoagulation and have excellent handling characteristics but may have lower immunogenicity and be more durable. This study analyzed our results of RVOT reconstruction using SGs and compared them with results of the SCAs used during the same period.

\section{PATIENTS AND METHODS Study Patients}

Between June 2000 and July 2005, 39 patients had implantation of a decellularized cryopreserved valve allograft (SynerGraft, SG; CryoLife) as a valved conduit for establishing continuity between the RV and PA at the Children's Hospital of Illinois, OSF Saint Francis Medical Center at Peoria. Sixty-one contemporary controls were included, who received standard cryopreserved pulmonary allografts (SCAs) during the same time period. The choice of conduit was made preoperatively or in the operating room by the surgeon on the basis of availability of allografts without randomization. This retrospective study was performed after the institutional review board approval was obtained.

All allografts were obtained from Cryolife, Inc (Marietta, Ga). No patient received a prosthesis that was undersized from a larger allograft. Blood group matching could not be accommodated owing to allograft availability. Conduit size was determined according to the calculated $Z$-value for each implanted valve using the valve diameter compared with the normal value. Our goal was to insert a conduit with a Z-score of +1 to +3 .

Demographic information, cardiac anatomy, preoperative hemodynamics, operative details, and postoperative outcomes were recorded retrospectively from patient records. Transthoracic echocardiography was used to evaluate conduit gradients and the degree of pulmonary insufficiency. Conduit stenosis was assessed by the measurement of peak velocity using continuous-wave Doppler technique. Valve regurgitation was quantified as trivial (grade 1), mild (grade 2, flow into the RV without flow reversal in the conduit), moderate (grade 3 , flow reversal in conduit), and severe (grade 4 , flow reversal in branch pulmonary arteries).

Graft dysfunction is considered to be present if the peak echocardiographic Doppler gradient is greater than $40 \mathrm{~mm} \mathrm{Hg}$ by 2-dimensional echocardiography at any level within the RVOT and/or grade 3 or 4 conduit valve insufficiency. Conduit failure is defined as the need for intervention in either the catheterization laboratory or operating room. Excluded are catheter interventions for peripheral PA stenosis not involving the distal conduit anastomosis. Indications for replacement of the homograft for conduit stenosis include a peak gradient greater than $40 \mathrm{~mm} \mathrm{Hg}$ and diminished $\mathrm{RV}$ function or RV pressures greater than $75 \%$ of systemic pressure. Indications for replacement for regurgitation include grade 3 or 4 insufficiency and evidence of progressive RV dilation by either echocardiography or magnetic resonance imaging.

The patient demographics are summarized in Table 1. There were no significant differences in age, number of neonates, sex, weight, previous surgery on the pulmonary valve, or preoperative diagnoses.

\section{Surgical Technique}

The standard approach includes median sternotomy, bicaval cardiopulmonary bypass, left ventricular venting, and moderate hypothermia $\left(28^{\circ} \mathrm{C}\right.$ $32^{\circ} \mathrm{C}$ ). Intracardiac repair was performed during aortic crossclamping with intermittent cold blood cardioplegia. Conduit insertion was most often performed with the crossclamp removed and the heart beating during rewarming. All allografts conduits were continuously agitated for 5 minutes in four 500-mL normal saline baths to remove glutaraldehyde. When applicable, the incision in the PA was extended leftward onto the left PA to accommodate a larger prosthesis and to avoid conduit distortion by the ascending aorta and the sternum. The outflow end of the allograft conduit was cut as short as possible to position the conduit valve at the distal anastomosis well to the left of the sternum. The proximal anastomosis was augmented anteriorly using a hood of polytetrafluoroethylene as needed. Stenosis in a branch PA was relieved most often by extension of the allograft onto the branch PAs.

The operative variables are summarized in Table 2. Concomitant procedures were not significantly different between the groups (SCA, 38/61, $62 \%$, vs SG, $20 / 39,51 \% ; P=.28$ ) and consisted of closure of atrial and ventricular septal defects, repair of tricuspid or mitral valves, patch enlargement or reduction of the PAs, and autograft replacement of the aortic valve (Ross procedure). The cardiopulmonary bypass time, cardiac ischemic times, and mean conduit size were similar.

\section{Follow-up}

All surviving patients were examined by serial transthoracic echocardiography every 6 to 12 months. Follow-up echo studies were available in $100 \%$ of patients. For SGs, the mean follow-up was $5.7 \pm 2.5$ years, ranging from 3 months to 10 years. The mean follow-up for SCAs was not significantly different at $5.8 \pm 2.8$ years, ranging from 3 months to 10 years $(P=.83)$. Early death was defined as death in the hospital or within 30 days of surgery. All other deaths were considered late.

\section{Statistical Analysis}

Data are presented as the mean \pm standard deviation. Continuous variables were analyzed with the Student $t$ test and categorical variables using the $\chi^{2}$ test. Variables for the 2 cohorts were compared using the 2-tailed unpaired $t$ test. Kaplan-Meier curves were generated for actuarial survival, freedom from conduit dysfunction, freedom from conduit failure, and freedom from conduit explantation. End points were time of death, first diagnosis of conduit dysfunction or failure, interventional or surgical reintervention, and conduit replacement. The log-rank test was used to estimate the statistical difference between the 2 types of conduits.

\section{RESULTS \\ Mortality}

There were 3 early deaths, 2 deaths in the SCA group and 1 death in SG group. A 2-weeks-old neonate with critical aortic stenosis and aortic insufficiency underwent RossKonno repair employing a 15-mm SCA for RVOT reconstruction and resection of endocardial fibroelastosis. This 
TABLE 1. Demographics

\begin{tabular}{|c|c|c|c|}
\hline & SG & SCA & $P$ value \\
\hline Age (mean, range) & $19.2 \pm 17.2(1 \mathrm{w}-72 \mathrm{y})$ & $19.4 \pm 16.2(1 \mathrm{w}-55 \mathrm{y})$ & .96 \\
\hline Infants $(<1$ year $)$ & $6(15 \%)$ & $17(28 \%)$ & .11 \\
\hline Weight (mean, range; kg) & $54.7 \pm 40.4(2.8-167)$ & $51.7 \pm 34.0(2.5-140)$ & .68 \\
\hline Gender (M/F) & $25 / 14$ & $33 / 28$ & .22 \\
\hline \multicolumn{4}{|l|}{ Preoperative diagnosis } \\
\hline AS (Ross procedure) & $16^{*}(41 \%)$ & $21 *(34 \%)$ & .53 \\
\hline Tetralogy of Fallot & $8(21 \%)$ & $15(25 \%)$ & .81 \\
\hline $\mathrm{PA} / \mathrm{VSD}$ & $7(18 \%)$ & $9(15 \%)$ & .78 \\
\hline Truncus arteriosus & $4(10 \%)$ & $3(5 \%)$ & .43 \\
\hline TGA & $2(5 \%)$ & $7(11 \%)$ & .48 \\
\hline Other & $2(5 \%)$ & $6(10 \%)$ & .48 \\
\hline Previous operation on PV & $20(51 \%)$ & $27(44 \%)$ & .32 \\
\hline
\end{tabular}

patient died 7 days postoperatively of sepsis and multiorgan system failure while receiving extracorporeal membrane oxygenation. The second death occurred in a 2-month-old neonate with transposition of great arteries, double-outlet $\mathrm{RV}$, pulmonary stenosis, and ventricular septal defect who underwent Rastelli repair with insertion of a 10-mm SCA. Twenty days later severe pulmonary regurgitation developed and the patient underwent redo surgery with replacement of the RV-PA conduit with a 12-mm Hancock xenograft (Medtronic, Inc, Minneapolis, Minn). The patient died on the third postoperative day of low cardiac output. The final death occurred in a 4-year-old patient with pulmonary atresia and ventricular septal defect who previously underwent RV-PA conduit insertion 2 times (SCA and Shelhigh conduits [Shelhigh, Inc, Union, NJ]). This patient underwent Rastelli-type repair with implantation of a 16-mm SG and died 1 week postoperatively of sepsis and multiorgan failure.

There were 4 SG and 4 SCA late deaths (SG, 4/38, $11 \%$, vs SCA, $4 / 59,7 \% ; P=.51)$. No death was attributed to

TABLE 2. Operative variables

\begin{tabular}{lccc}
\hline & SG & SCA & $P$ value \\
\hline Additional cardiac procedures & $20(51 \%)$ & $38(62 \%)$ & .28 \\
Pulmonary autograft & 12 & 18 & \\
PA branch arterioplasty & 3 & 10 & \\
TV repair & 2 & 6 & \\
ARR & 2 & 2 & \\
IAA repair & 1 & 1 & \\
CAVC repair & 0 & 1 & \\
CPB (min) & $175 \pm 103$ & $181 \pm 68$ & .76 \\
Aortic crossclamping time (min) & $95 \pm 62$ & $104 \pm 68$ & .50 \\
Diameter of conduit (min, mm) & $21.0 \pm 4.2$ & $20.6 \pm 6.2$ & .69 \\
Range (mm) & $10-27$ & $8-28$ & \\
\hline
\end{tabular}

$S G$, SynerGraft; $S C A$, standard cryopreserved allograft; $P A$, pulmonary artery; $T V$, tricuspid valve; $A R R$, aortic root replacement; $I A A$, interrupted aortic arch; $C A V C$, complete atrioventricular canal; $C P B$, cardiopulmonary bypass. structural failure of the conduit. The actuarial survival including hospital deaths for SG and SCAs at 5 (SG, 92\%; SCA, 89\%) and 10 years (SG, 90\%; SCA, 87\%) was similar for both groups $(P=.75)$ (Figure 1). The causes of late deaths were low-output cardiac failure in patients with congenital noncardiac and cardiac anomalies $(\mathrm{n}=3)$, pneumonia $(\mathrm{n}=2)$, noncardiac $(\mathrm{n}=2)$, and unknown $(\mathrm{n}=1)$.

\section{Freedom From Conduit Dysfunction}

Among the 97 hospital survivors, 38 patients (SG, 10/38, $26 \%$; SCA, 28/59, 52\%) had evidence of significant conduit dysfunction at the most recent follow-up or before conduit replacement. The mode of dysfunction was characterized as follows: stenosis with regurgitation in 22 patients and stenosis or insufficiency alone in 10 and 6 patients, respectively. Freedom from conduit dysfunction was significantly worse in the SCA group $(69 \%$ and $48 \%$ at 5 and 10 years, respectively) as compared with the SG group $(82 \%$ and $74 \%$ at 5 and 10 years, respectively; $P=.05)$ (Figure 2).

\section{Freedom From Conduit Failure}

At latest follow-up, 5 (13\%) patients in the SG group exhibited conduit failure secondary to stenosis and/or valve dysfunction: 3 patients, explantation; 1 patient, percutaneous intervention; and 1 patient for both. The mean time to reoperation in the SG group was $3.4 \pm 3.1$ years (range, 9 months to 8 years). Percutaneous interventions were required in 2 patients (balloon dilatation, 1 patient; stent implantation, 1 patient); of these, 1 patient required conduit explantation at 2 years after intervention.

Conduit failure was observed in $19(33 \%)$ of 58 SCA patients (all surgical interventions). All surgical procedures were explants. The mean time to reoperation for SCA was $3.4 \pm 2.8$ years (range, 2 months to 9 years) and was not significantly different from the SG group $(P=.96)$. At 5 and 


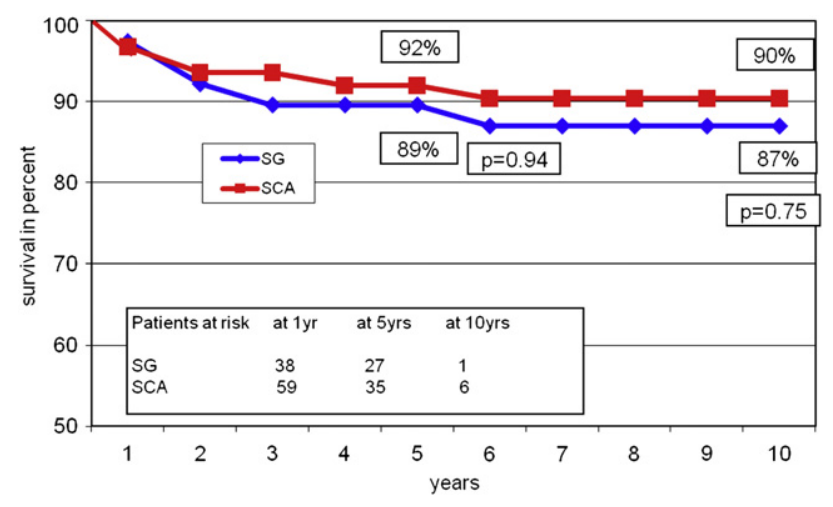

FIGURE 1. Kaplan-Meier estimated 10-year survival, including hospital mortality. $S G$, SynerGraft; $S C A$, standard cryopreserved allograft.

10 years after implantation, the freedom from conduit failure was significantly higher in the SG group $(90 \%$ and $87 \% ; P=.05$; Figure 3 ). Freedom from explantation at 5 and 10 years was also significantly higher in the SG group $(92 \%$ and $90 \% ; P=.02$; Figure 4$)$. The freedom from conduit failure was significantly higher in the SCA group for patients with small conduits $(\leq 19 \mathrm{~mm}$; SCA $13 \%$ vs SG $64 \% ; P=.01$ ) but not significant different for patients with bigger conduits $(\geq 20 \mathrm{~mm}$; SCA $86 \%$ vs SG $93 \%$; $P=.23$ ).

\section{Conduit Retention and Function at Last Follow-up}

Among the 89 late survivors (SG, 34/39, 87\%, vs SCA, $55 / 61,90 \% ; P=.75), 65$ patients retained their initial conduit at last follow-up (SG, 29/34, 85\%, vs SCA, 36/55, $66 \% ; P=.05)$. In the $\mathrm{SG}$ group, pulmonary regurgitation was trivial in $13(45 \%)$ patients, mild in $13(45 \%)$, and moderate in $3(10 \%)$ patients. Two patients had transconduit gradients above $40 \mathrm{~mm} \mathrm{Hg}(58$ and $64 \mathrm{~mm} \mathrm{Hg}$ ). Conduit regurgitation among the 36 SCA patients was trivial in $16(44 \%)$, mild in $15(42 \%)$, and moderate in $5(14 \%)$ patients. At latest follow-up, 4 (11\%) patients in the SCA group were being followed up with a transconduit gradient

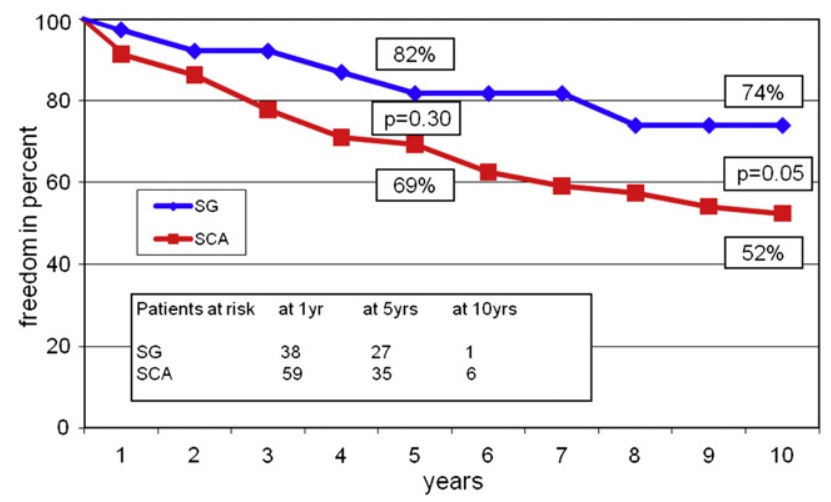

FIGURE 2. Kaplan-Meier estimated 10-year freedom from conduit dysfunction. $S G$, SynerGraft; $S C A$, standard cryopreserved allograft.

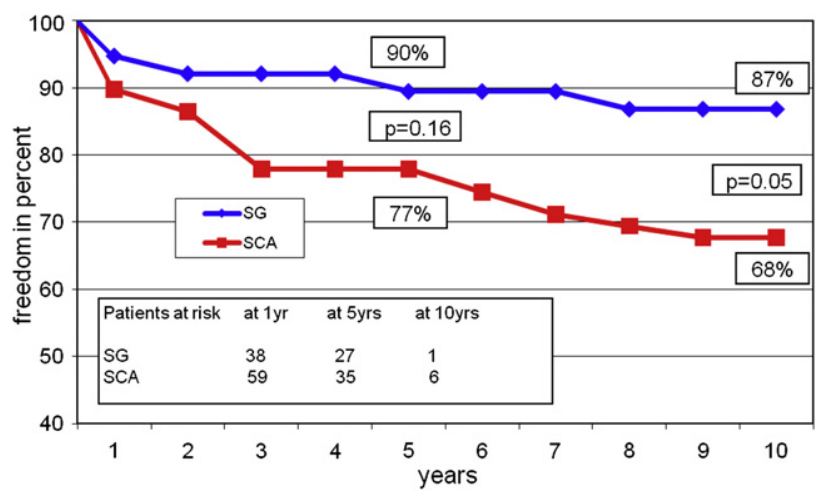

FIGURE 3. Kaplan-Meier estimated 10-year freedom from conduit failure. $S G$, SynerGraft; $S C A$, standard cryopreserved allograft.

above $40 \mathrm{~mm} \mathrm{Hg}(41,49,52$, and $64 \mathrm{mmHg}$ ), including 1 patient with moderate pulmonary regurgitation.

\section{Conduit Outcome in Patients Less Than 1 Year of Age}

We also compared the performance of conduits inserted primarily in patients less than 1 year of age ( $\mathrm{SG}, \mathrm{n}=6$; SCA, $n=17$ ). Survival was $67 \%$ in the SG group and $71 \%$ in the SCA group $(P=.85)$. Freedom from explantation (SG $67 \%$ vs SCA $25 \% ; P=.10$ ) and failure (SG $50 \%$ vs SCA $25 \% ; P=.27)$ trended lower in the SG cohort but did not reach statistical significance. Freedom from conduit dysfunction also trended lower in the SG cohort (SG 50\% vs SCA $19 \% ; P=.14)$.

\section{COMMENT}

Reconstructing RV-PA continuity using valved conduits is an essential component in the treatment of many patients with congenital heart disease. The valved allograft first reported in the late 1960s has been the principal conduit used in the United States since the mid-1980s. However, midterm and long-term follow-up studies have clearly demonstrated conduit obstruction and early valve insufficiency. $^{2-4}$ In the majority of reports, all patients have

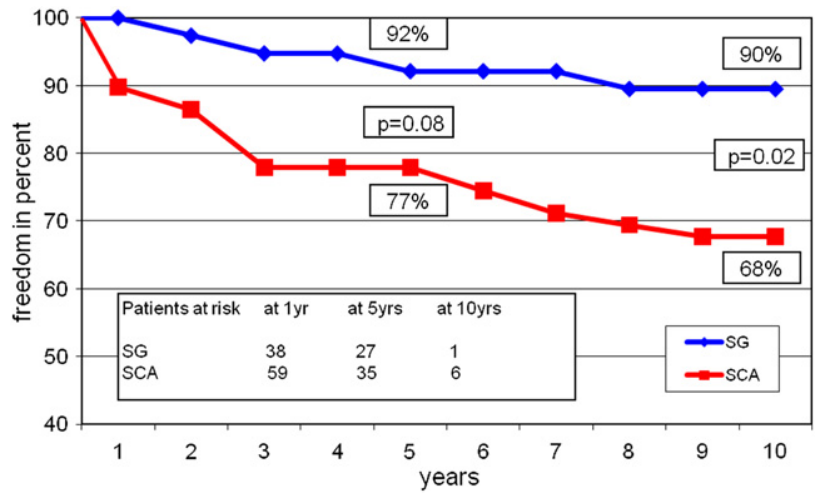

FIGURE 4. Kaplan-Meier estimated 10-year freedom from conduit explantation. $S G$, SynerGraft; $S C A$, standard cryopreserved allograft. 
some conduit valve regurgitation at or before 1 year. ${ }^{5,8}$ Additionally, virtually all allografts calcify, but the rate of calcification appears significantly greater in aortic allografts, perhaps because of high elastin tissue content within the aortic conduit wall. ${ }^{5}$ Accelerated degeneration has been observed for aortic and pulmonary allograft conduits implanted in patients less than 1 year of age, perhaps owing to host immunologic response to viable endothelial cells within some SCA conduits. ${ }^{4-6}$

In addition, there is growing concern over donor-specific cellular responses and elevated levels of tissue-specific antibodies found in allograft valve recipients. Host antigen recognition and antibody development may be linked to early-onset tissue calcification and structural valve deterioration. ${ }^{8,10,11}$ High panel reactive antibody (PRA) levels may decrease the number of potential donors for those patients who progress to a need for transplantation. ${ }^{10}$ Vogt and colleagues ${ }^{12}$ identified cellular rejection in the wall of allograft material explanted from the RVOT of children. These inflammatory cells were predominantly $\mathrm{T}$ lymphocytes. Rajani, Mee, and Ratliff ${ }^{9}$ identified cellular rejection in the wall of allograft material explanted from the RVOT of children, and the inflammatory cells identified were predominantly $\mathrm{T}$ lymphocytes. Shaddy and associates ${ }^{13}$ prospectively measured the frequency of human leukocyte antigen class I PRA in children undergoing open cardiac operations with or without the implantation of allograft material. They found that the PRA in patients receiving an SCA increased within months of the operation, whereas PRA did not change significantly in the control group. This immune response is believed to have been caused by the SCA and may be a contributory factor in the early degradation of allograft function in some patients.

SG technology was developed as a decellularization process for allograft tissue to reduce antigenicity. SG methods result in native cell removal from the collagen tissue matrix and the endothelium. Removal of cellular material may reduce or eliminate the immunologic response and leave a functional vascular matrix that is available for autogenous remodeling. Migration of the recipient-specific cells into the matrix may eventually render the graft indistinguishable from other endogenous tissue. ${ }^{14}$ Elkins and colleagues ${ }^{11}$ demonstrated the preservation of tissue strength, biomechanics, and valvular hydrodynamic function. The SG pulmonary valve conduit was introduced into clinical use in 2000.

An in vitro comparison of decellularized human SGs with SCAs revealed similar tissue mechanics and hemodynamic performance. Hemodynamic testing revealed no differences in valve gradient, valve closure volume, or diastolic regurgitation. ${ }^{11,15}$ Uniaxial tension loading demonstrated no difference in conduit failure, leaflet failure, or suture retention. ${ }^{15}$ Alfonso da Costa and colleagues ${ }^{16}$ demonstrated that decellularized allografts are less antigenic than standard cryopreserved allografts and exhibited normal hemodynamic performance in the right side of the circulation up to 18 months after implantation. In contrast to the SCAs, they could not detect any elevation in antibody and PRA levels in 7 cases and only marginal elevation in 2 others. $^{16}$

Tavakkol and associates ${ }^{15}$ demonstrated the superior short-term durability of the SGs compared with SCAs with respect to both stenosis and insufficiency. At longer follow-up, Konuma and colleagues ${ }^{10}$ (from the same center) found there were no significant differences between SGs compared with SCA conduits with reference to stenosis and valve insufficiency, although the overall degree of insufficiency was higher in the SCAs $(P=.07)$. However, when they analyzed the incidence of clinically important $(>3+)$ insufficiency, they found a statistically significant advantage for the SG valve. ${ }^{10}$

Bechtel and colleagues ${ }^{17,18}$ evaluated a cohort of adult patients who had undergone the Ross procedure for performance of SGs in the RVOT in comparison with SCAs. They demonstrated no difference with regard to conduit regurgitation or reintervention, but surprisingly SCAs were superior to SGs with regard to conduit stenosis. Burch and colleagues ${ }^{19}$ concluded that decellularized SGs have a nonsignificant trend toward lower peak valve gradients and fewer reinterventions compared with SCAs. Small valve sizes $(\leq 18 \mathrm{~mm})$ show a slight but significant improvement in peak gradient but no advantage in valve insufficiency.

Brown and colleagues ${ }^{20}$ previously reported multicenter results comparing SG and SCA RV-PA conduits in children after a Ross procedure and demonstrated no difference in RVOT gradient but did note a significant reduction of midterm regurgitation in the SG groups. ${ }^{20}$ This multicenter, retrospective cohort study of 342 consecutive patients who received the SG supported earlier evidence that the SG is an appropriate graft for patients requiring pulmonary valve replacement or reconstruction. Overall, at a mean of 4 years, the SG appears to be safe and has at least comparable, if not improved, hemodynamic performance. ${ }^{20}$

In conclusion, our study suggests that the midterm performance of SGs may be superior to that of SCAs. Decellularization of the cryopreserved allografts may provide a more durable option for patients who need RVOT reconstruction. Further long-term follow-up is needed to see whether this decellularization process improves long-term allograft durability.

A number of important limitations are present in this analysis. Data were acquired retrospectively and in a nonrandomized manner. The operations were performed by 2 surgeons and the decision for conduit type was determined by the patient's anatomy and the conduit's availability, not by randomization. Longer follow-up, larger numbers of patients, matched cohorts, and multicenter prospective study 
are necessary to further evaluate the performance of the decellularized cryopreserved allograft valves in the pulmonary position.

\section{References}

1. Brown JW, Ruzmetov M, Eltayeb O, Rodefeld MD, Turrentine MW. Performance of SynerGraft decellularized pulmonary homograft in patients undergoing a Ross procedure. Ann Thorac Surg. 2011;91:416-23.

2. Forbess JM, Shah AS, St Louis JD, Jaggers JJ, Ungerleider RM. Cryopreserved homografts in the pulmonary position: determinants of durability. Ann Thorac Surg. 2001;71:54-60.

3. Dearani JA, Danielson GK, Puga FJ, Schaff HV, Warnes CW, Driscoll DJ, et al. Late follow-up of 1095 patients undergoing operation for complex congenital heart disease utilizing pulmonary ventricle to pulmonary artery conduits. Ann Thorac Surg. 2003;75:399-411.

4. Brown JW, Ruzmetov M, Rodefeld MD, Vijay P, Turrentine MW. Right ventricular outflow tract reconstruction with an allograft conduit in non-Ross patients: risk factors for homograft dysfunction and failure. Ann Thorac Surg. 2005;80:655-64.

5. Wells WJ, Arroyo H Jr, Bremner RM, Wood J, Starnes VA. Homograft conduit failure in infants is not due to somatic outgrowth. $J$ Thorac Cardiovasc Surg. 2002;124:88-96.

6. Bielefeld MR, Bishop DA, Campbell DN, Mitchell MB, Grover FL, Clarke DR. Reoperative homograft right ventricular outflow tract reconstruction. Ann Thorac Surg. 2001;71:482-8.

7. Hawkins JA, Hillman ND, Lambert LM, Jones J, Di Russo GB, Profaizer T, et al. Immunogenicity of decellularized cryopreserved allografts in pediatric cardiac surgery: comparison with standard cryopreserved allografts. J Thorac Cardiovasc Surg. 2003;126:247-53.

8. Yankah AC, Alexi-Meskhishvili V, Weng Y, Schorn K, Lange PE, Hetzer R. Accelerated degeneration of allografts in the first two years of life. Ann Thorac Surg. 1995;60:S71-7.

9. Rajani B, Mee RB, Ratliff NB. Evidence for rejection of homograft cardiac valves in infants. $J$ Thorac Cardiovasc Surg. 1998;115:111-7.

10. Konuma T, Devaney EJ, Bove EL, Gelehrter S, Hirsch JC, Tavakkol Z, et al. Performance of CryoValve SG decellularized pulmonary allografts compared with standard cryopreserved allografts. Ann Thorac Surg. 2009;88:849-55.

11. Elkins RC, Dawson PE, Goldstein S, Walsh SP, Black KS. Decellularized human valve allografts. Ann Thorac Surg. 2001;71(5 suppl):S428-32.

12. Vogt PR, Stallmach T, Niederhäuser U, Schneider J, Zünd G, Lachat M, et al. Explanted cryopreserved allografts: a morphological and immunohistochemical comparison between arterial allografts and allograft heart valves from infants and adults. Eur J Cardiothorac Surg. 1999;15:639-45.

13. Shaddy RE, Hunter DD, Osborn KA, Lambert LM, Minich LL, Hawkins JA, et al. Prospective analysis of HLA immunogenicity of cryopreserved valved allografts used in pediatric heart surgery. Circulation. 1996;94:1063-7.

14. Clarke DR, Campbell DN, Hayward AR, Bishop DA. Degeneration of aortic valve allografts in young recipients. J Thorac Cardiovasc Surg. 1993;105:934-41.

15. Tavakkol Z, Gelehrter S, Goldberg CS, Bove EL, Devaney EJ, Ohye RG. Superior durability of Synergraft pulmonary allografts compared with standard cryopreserved allografts. Ann Thorac Surg. 2005;80:1610-4.

16. Alfonso da Costa FD, Dohmen PM, Duarte D, von Glenn C, Lopes SV, Filho HH, et al. Immunological and echocardiographic evaluation of decellularized versus cryopreserved allografts during the Ross operation. Eur J Cardiothorac Surg. 2005;27:572-8.

17. Bechtel JFM, Stierle U, Sievers H- H. Fifty-two monthes' mean follow-up of decellularized SynerGraft-treated pulmonary valve allografts. $J$ Heart Valve Dis. 2008;17:98-104.

18. Bechtel JF, Gellissen J, Erasmi AW, Petersen M, Hiob A, Stierle U, et al. Mid-term findings on echocardiography and computed tomography after RVOT-reconstruction: comparison of decellularized (SynerGraft) and conventional allografts. Eur J Cardiothorac Surg. 2005;27:410-5.

19. Burch PT, Kaza AK, Lambert LM, Holubkov R, Shaddy RE, Hawkins JA. Clinical performance of decellularized cryopreserved valved allografts compared with standard allografts in the right ventricular outflow tract. Ann Thorac Surg. 2010;90:1301-6.

20. Brown JW, Elkins RC, Clarke DR, Tweddell JS, Huddleston CB, Doty JR, et al. Performance of the CryoValve SG human decellularized pulmonary valve in 342 patients compared to the conventional CryoValve at a mean follow-up of 4 years. J Thorac Cardiovasc Surg. 2010;139:339-48.

\section{Discussion}

Dr James Jaggers (Aurora, Colo). Dr Fortuna and his colleagues have presented today a single-institution series of 100 patients over 7 years that underwent RVOT reconstruction with 39 of them receiving an SG and 61 receiving an SCA. Importantly, Dr Fortuna, you have shown that SG valves had superior performance with statistically better freedom from conduit failure, conduit dysfunction, and conduit replacement at 10 years. This should be noted to be in contrast with previous experience that showed a very limited improvement in outcomes with the SG, most of those related to mild improvement and regurgitation. As you noted, important limitations of your study and others like this one regarding its nonrandomized nature, its retrospective nature, and important differences in the 2 groups leave the audience to wonder whether these patient groups are really the same. For example, there are quite a few more infants receiving smaller allografts in the SCA group versus the SG group.

I just have 3 brief questions for you. First of all, when you mentioned that the echocardiographic analysis was done by a single interpreter, was this interpretation done in a prospective manner or were these based on previous descriptions in their echocardiographic reports?

Dr Fortuna. Thank you. We obtained the original echocardiographic studies and presented them to a single reviewer in a blinded fashion. This person reviewed and graded them in a standardized fashion.

Dr Jaggers. Importantly, did you find any difference when you analyzed your data between patients who received the orthotopic versus the heterotopic implant? For example, it is generally recognized that Ross procedure patients have better performance of their allografts than non-Ross patients. Did you have a chance to look at your data that way?

Dr Fortuna. I think that is a very good question. It has been shown at multiple institutions that allografts are more durable when placed in an orthotopic position versus a heterotopic position. In our group we were not able to show any difference.

Dr Jaggers. If you could speculate on the modes of failure, did you find that the SGs have less calcification? Is there anything that you can determine that would explain why SGs have improved performance?

Dr Fortuna. In this retrospective study we did not collect the tissue samples or systematically study them, but our general impression is that there may be less calcification in the SGs as opposed to the SCAs. It is interesting that the function of the valve seems to be most dramatically affected, and in that case the calcification may play a significant role in the function of those leaflets.

Dr Jaggers. Finally, because these valves are of limited availability, in your clinical experience, is there a particular patient group in which you would elect to use an SG versus an SCA?

Dr Fortuna. In our institution our bias is to use the SG in all patients if we have that opportunity. I think it is a very valid point that since other studies have shown some risk factors for conduit failure such as you mentioned, the heterotopic position of the conduit, maybe those patients may benefit most from the SG technology. We are currently in the process of combining our institution's data with those of several other institutions, and our hopes are that by combining all of that information we will be able to do a more robust statistical analysis and perhaps do some subgroup analysis 
as well to provide more information about who would benefit most.

Dr Jaggers. I enjoyed your paper very much and I think it is going to be a valuable contribution.

Dr Fortuna. Thank you.

Speaker. I have a question. You are implying that you have 10year data, but if I looked at your slides right, at 10 years you had 1 SG patient and 5 SAG patients. Is that correct?

Dr Fortuna. Yes.

Speaker. Do you really consider that to be valid 10-year data.

Dr Fortuna. No, I am not sure that is an entirely valid comparison out at 10 years because of the small numbers, but it does reach statistical significance. Also, I think you get the sense from looking at those Kaplan-Meier graphs that there is a divergence in outcomes between the SGs and the SCAs. Only with longer follow up will we know whether that maintains statistical significance.
Speaker. I agree with the early part of your discussion.

Did you make any effort to propensity match these groups, and don't you think that would make your data much more believable?

Dr Fortuna. That is a very valid point. We may have been able to do that. However, we thought there was some value in keeping a contemporary group for comparison because of other unknown factors that may affect outcomes. If we did propensity matching with patients who are taken out of other time periods, for example, it might be difficult, albeit possible, to take some of those other factors into consideration. We thought it was best in a single-institution experience with a limited number of patients to take the contemporary group in hopes that we are eliminating some of those other factors.

Speaker. I suggest it may be possible to propensity match even within your own group, but congratulations on the study.

Dr Fortuna. Thank you. 